\title{
Podophyllotoxin Induces ROS-Mediated Apoptosis and Cell Cycle Arrest in Human Colorectal Cancer Cells via p38 MAPK Signaling
}

\author{
Seung-On Lee ${ }^{1, \dagger}$, Sang Hoon Joo ${ }^{2, \dagger}$, Ah-Won Kwak ${ }^{3}$, Mee-Hyun Lee ${ }^{4}$, Ji-Hye Seo ${ }^{5}$, Seung-Sik Cho ${ }^{3}$, Goo Yoon ${ }^{3}$, \\ Jung-II Chae ${ }^{5, *}$ and Jung-Hyun Shim ${ }^{1,3,6, *}$
}

${ }^{1}$ Department of Biomedicine, Health \& Life Convergence Sciences, BK21 Four, Biomedical and Healthcare Research Institute, Mokpo National University, Muan 58554,

${ }^{2}$ College of Pharmacy, Daegu Catholic University, Gyeongsan 38430,

${ }^{3}$ Department of Pharmacy, College of Pharmacy, Mokpo National University, Muan 58554 ,

${ }^{4}$ College of Korean Medicine, Dongshin University, Naju 58245,

${ }^{5}$ Department of Dental Pharmacology, School of Dentistry, Jeonbuk National University, Jeonju 54896, Republic of Korea

${ }^{6}$ The China-US (Henan) Hormel Cancer Institute, Zhengzhou, Henan 450008, China

\begin{abstract}
Podophyllotoxin (PT), a lignan compound from the roots and rhizomes of Podophyllum peltatum, has diverse pharmacological activities including anticancer effect in several types of cancer. The molecular mechanism of the anticancer effects of PT on colorectal cancer cells has not been reported yet. In this study, we sought to evaluate the anticancer effect of PT on human colorectal cancer HCT116 cells and identify the detailed molecular mechanism. PT inhibited the growth of cells and colony formation in a concentration-dependent manner and induced apoptosis as determined by the annexin V/7-aminoactinomycin D double staining assay. PT-induced apoptosis was accompanied by cell cycle arrest in the G2/M phase and an increase in the generation of reactive oxygen species (ROS). The effects of $\mathrm{PT}$ on the induction of ROS and apoptosis were prevented by pretreatment with $\mathrm{N}$-acetyl-L-cysteine (NAC), indicating that an increase in ROS generation mediates the apoptosis of HCT116 cells induced by PT. Furthermore, Western blot analysis showed that PT upregulated the level of phospho (p)-p38 mitogen-activated protein kinase (MAPK). The treatment of SB203580, a p38 inhibitor, strongly prevented the apoptosis induced by PT, suggesting that PT-induced apoptosis involved the p38 MAPK signaling pathway. In addition, PT induced the loss of mitochondrial membrane potential and multi-caspase activation. The results suggested that PT induced cell cycle arrest in the G2/M phase and apoptosis through the p38 MAPK signaling pathway by upregulating ROS in HCT116 cells.
\end{abstract}

Key Words: Podophyllotoxin, Colon cancer, Cell cycle arrest, Reactive oxygen species, p38, Apoptosis

\section{INTRODUCTION}

Colorectal cancer is a highly prevalent cancer, and the second leading cause of cancer-related death worldwide (Sung et al., 2021). Currently, the approaches to treating patients with colorectal cancer are surgery, radiotherapy, and chemotherapy (Xie et al., 2020). Despite the advanced therapies for colorectal cancer, the 5-year survival rate of patients remains at only $64 \%$ (Siegel et al., 2020). The first-line treatment for patients with colorectal cancer is chemotherapy based on 5 -fluorouracil, but it has limitations including a low response rate, system- ic toxicity, and low selectivity for cancer cells (Xie et al., 2020). Therefore, new and more effective anticancer drug candidates for patients with colorectal cancer are required to improve the treatment outcomes and reduce adverse side effects.

Podophyllotoxin (PT) is a natural lignan derived from the roots and rhizomes of Mayapple Podophyllum peltatum, a perennial herb distributed widely across the Himalayan region and Western China (Canel et al., 2000; Shah et al., 2021). Podophyllum has long been used as a drug in different countries for multiple purposes including as an emetic, cathartic, and for the treatment of venereal warts (Shah et al., 2021).

\section{Open Access https://doi.org/10.4062/biomolther.2021.143}

This is an Open Access article distributed under the terms of the Creative Commons Attribution Non-Commercial License (http://creativecommons.org/licenses/by-nc/4.0/) which permits unrestricted non-commercial use, distribution, and reproduction in any medium, provided the original work is properly cited.
Received Aug 31, 2021 Revised Sep 9, 2021 Accepted Sep 10, 2021 Published Online Oct 13, 2021

\section{*Corresponding Authors}

E-mail: s1004jh@gmail.com (Shim JH), jichae@jbnu.ac.kr (Chae JI) Tel: +82-61-450-2684 (Shim JH), +82-63-270-4024 (Chae Jl) Fax: +82-61-450-2689 (Shim JH), +82-63-270-4037 (Chae JI) ${ }^{\dagger}$ The first two authors contributed equally to this work. 
The pharmacological effects of PT include anti-malaria, antirheumatic, anti-viral, and anticancer activity (Hu et al., 2020). Current studies suggest that PT exerts antitumor effects by inhibiting DNA topoisomerase II, which plays an important role in DNA replication (Sathish et al., 2018). In addition, PT prevents microtubule assembly by inhibiting tubulin polymerization, arresting the cell cycle (Sathish et al., 2018). Etoposide, a derivative of PT, was granted Food and Drug Administration approval and has been used for treating a variety of cancer types, such as testicular cancer and small cell lung cancer (Hande, 1998).

Apoptosis, which is programmed cell death, is essential for normal tissue homeostasis (Qiao and Wong, 2009). Two signaling pathways are involved in activating apoptosis, the extrinsic and intrinsic pathways (Khan et al., 2014). The extrinsic pathway is mediated by tumor necrosis factor-related apoptosis-inducing ligand receptor 1 (TRAIL-R1, DR4) and TRAILR2 (DR5), resulting in the activation of caspase-8, which is the initiator caspase (Moradi Marjaneh et al., 2018). In the intrinsic pathways, the $\mathrm{Bcl}-2$ family proteins play an important role in determining whether cells will undergo apoptosis. Shifting the balance of pro-apoptotic and anti-apoptotic $\mathrm{Bcl}-2$ proteins can lead to cytochrome $c$ release from the mitochondria followed by the activation of caspase-3 and -9 (Deng et al., 2000; Khan et al., 2014). Evasion from apoptosis can be a survival strategy of cancer cells (Deng et al., 2000), and high levels of antiapoptotic Bcl-2 proteins would allow cancer cells to survive (Reed et al., 1988). Conversely, restoration of the apoptotic pathway in cancer cells could be a promising approach to cancer therapy.

Reactive oxygen species (ROS) including superoxide, the hydroxyl radical, and hydrogen peroxide are highly reactive molecules derived from oxygen (Martindale and Holbrook, 2002). ROS induce the activation of the mitogen-activated protein kinase (MAPK) signaling pathway involved in cellular processes, such as stress adaptation, differentiation, cell growth, proliferation, and apoptosis (Martindale and Holbrook, 2002; Ko et al., 2019). Due to the beneficial roles of ROS involved in proliferation and apoptosis, cancer cells usually maintain high levels of ROS (Schieber and Chandel, 2014; Choi et al., 2021). Ironically, high levels of ROS accumulation in cancer cells can be exploited: cellular oxidative stress-induced apoptosis of cancer cells (Nogueira and Hay, 2013). Thus, increasing ROS levels in cancer cells to toxic levels is considered a powerful strategy to specifically target cancer cells. The p38 pathway, a subfamily of MAPK signaling, is one of the stress response pathways activated by increased ROS levels. The activation of the p38 pathway can result in the induction of apoptotic cell death (Osone et al., 2004; Wang et al., 2013).

The molecular mechanism of the anticancer effects of PT on colorectal cancer cells has not been reported. This study was conducted to evaluate the anticancer effect of PT on colorectal cancer and determine its apoptotic mechanism related to ROS and p38 MAPK.

\section{MATERIALS AND METHODS}

\section{Reagents}

Roswell Park Memorial Institute (RPMI)-1640 medium, fetal bovine serum (FBS), trypsin, penicillin/streptomycin ( $\mathrm{p} / \mathrm{s})$, and phosphate-buffered saline (PBS), were from Hyclone (Lo- gan, UT, USA). podophyllotoxin (PT; purity greater than 98\%), dimethyl sulfoxide (DMSO), 3-(4,5-dimethyl-2-thiazolyl)-2,5diphenyl-2H-tetrazolium bromide (MTT), N-acetylcysteine (NAC), basal medium eagle (BME), carbobenzoxy-valyl-alanyl-aspartyl-(O-methyl)-fluoromethylketone (Z-VAD-FMK), and p38 inhibitor (SB203580) were procured from Sigma Aldrich (St. Louis, MO, USA). Antibodies specific to cyclin B1, cdc2, $\beta$-actin, p21, p27, 78-kDa glucose-regulated protein (GRP78), $\mathrm{C} / \mathrm{EBP}$ homologous protein (CHOP), death receptor (DR)4, DR5, apoptotic protease-activating factor-1 (Apaf-1), Bid, $\mathrm{Bcl}-\mathrm{xl}, \mathrm{Mcl}-1$, Bad, caspase 3 and horseradish peroxidaseconjugated secondary antibodies (goat-anti-rabbit, goat-antimouse) were obtained from Santa Cruz Biotechnology (Santa Cruz, CA, USA). Antibodies against phosphor (p)-p38 MAPK (Thr180/Tyr182) and p38 were purchased from Cell Signaling Technology (Beverly, MA, USA).

\section{Cell culture and PT treatment}

Human colon cancer HCT116 cells were obtained from the American Type Culture Collection (ATCC, Manassas, VA, USA), and maintained in RPMI-1640 medium containing 10\% FBS and $100 \mathrm{U} / \mathrm{mL}$ penicillin/streptomycin. The cells were incubated at $37^{\circ} \mathrm{C}$ in a $5 \% \mathrm{CO}_{2}$ humidified incubator. To determine the effect of PT on HCT116 cells, the cells were treated with varying concentrations of $\mathrm{PT}(0,0.1,0.2$, and $0.3 \mu \mathrm{M})$ for 24 or $48 \mathrm{~h}$. DMSO was used as the vehicle.

\section{MTT (3-(4,5-dimethylthiazol-2-yl)-2,5- diphenyltetrazolium bromide) assay}

The MTT assay was used to determine cell viability. HCT116 cells were seeded in a 96 -well plate $(5,000$ cells/well) and treated with PT the next day. After 24 or $48 \mathrm{~h}$ of treatment with PT, MTT reagent was added to each well $(0.5 \mathrm{mg} /$ $\mathrm{mL}$ final concentration) for $1 \mathrm{~h}$ incubation at $37^{\circ} \mathrm{C}$. Then, the formazan crystals were dissolved by adding $100 \mu \mathrm{L}$ of DMSO to each well. The absorbance of the samples was measured at $570 \mathrm{~nm}$ using a Multiskan GO spectrophotometer (Thermo Scientific, Vantaa, Finland) to assess cell viability. The relative cell viability was calculated compared to the negative controls.

\section{Soft agar assay}

The soft agar assay was used to evaluate anchorage-independent growth. For the bottom layer, $1 \mathrm{~mL}$ of $0.6 \%$ agar was poured into each well of a 6 -well plate with culture medium and PT in DMSO, and allowed to solidify. Then, the upper layers $\left(8 \times 10^{3}\right.$ cells/well) were seeded with culture medium containing $0.3 \%$ agar and PT in DMSO. Seven days later, microscopic observation was done to measure the size and number of colonies (Leica Microsystems, Wetzlar, Germany).

\section{Annexin V/7-aminoactinomycin D (7-AAD) double staining assay}

To detect HCT116 cell apoptosis, the Muse ${ }^{\mathrm{TM}}$ Annexin V \& Dead Cell Kit (MCH100105, EMD Millipore, Billerica, MA, USA) was used following the manufacturer's instructions. Briefly, cells treated with PT for $48 \mathrm{~h}$ were harvested and washed in 1xphosphate-buffered saline (PBS), and stained with the Muse ${ }^{\mathrm{TM}}$ Annexin $\mathrm{V}$ \& Dead Cell reagents (EMD Millipore). The stained cells were incubated at room temperature (RT) for $20 \mathrm{~min}$ in the dark. Fluorescence intensity was measured by flow cytometry using a Muse ${ }^{\mathrm{TM}}$ Cell Analyzer (EMD Millipore), and the cells were sorted into live (annexin 
V-/7-AAD-), early apoptotic (annexin $V+/ 7-A A D-)$, late apoptotic (annexin $\mathrm{V}+/ 7-A A D+$ ), and necrotic (annexin $\mathrm{V}-/ 7-A A D+$ ) cells. The sum of the early and late apoptotic cells was calculated as the total number of apoptotic cells.

\section{Cell cycle analysis}

For cell cycle analysis, cells treated with PT were harvested, washed with ice-cold $1 \times$ PBS, and $70 \%$ ethanol was added for fixing. The cells were incubated overnight at $-20^{\circ} \mathrm{C}$, and then the fixed cells were collected by centrifugation at 4,000 rpm for $10 \mathrm{~min}$ and rinsed with $1 \times$ PBS. The cell pellet was resuspended in Muse ${ }^{\mathrm{TM}}$ Cell Cycle Reagent (MCH100106, EMD Millipore), and the cells were incubated for $30 \mathrm{~min}$ at RT in the dark. The DNA content was assessed by measuring the fluorescence intensity by flow cytometry (Muse ${ }^{\mathrm{TM}}$ Cell Analyzer).

\section{Measurement of intracellular ROS levels}

The Muse ${ }^{\mathrm{TM}}$ Oxidative Stress kit (EMD Millipore) was used to measure intracellular ROS levels. In brief, cells treated with PT were harvested and washed with $1 \times$ assay buffer, and then incubated with Muse ${ }^{\mathrm{TM}}$ Oxidative Stress Reagent working solution (MCH100111, EMD Millipore) at $37^{\circ} \mathrm{C}$ for $30 \mathrm{~min}$. The ROS levels were determined by flow cytometry (Muse ${ }^{\mathrm{TM}}$ Cell Analyzer).

\section{Measurement of mitochondrial membrane potential (MMP)}

To assess the changes in the mitochondrial membrane potential (MMP) in PT-treated cells, the cells were stained with MitoPotential dye and 7-AAD using the Muse ${ }^{\mathrm{TM}}$ MitoPotential Kit (MCH100110, EMD Millipore). Briefly, cells treated with PT were harvested and washed with $1 \times$ assay buffer. The washed cells were resuspended in Muse ${ }^{\mathrm{TM}}$ MitoPotential working solution and incubated for $20 \mathrm{~min}$ at $37^{\circ} \mathrm{C}$. Muse ${ }^{\mathrm{TM}}$ MitoPotential 7-AAD reagent was added for $5 \mathrm{~min}$ of incubation at RT, then changes in the MMP were determined by flow cytometry (Muse ${ }^{\mathrm{TM}}$ Cell Analyzer).

\section{Measurement of multi-caspase activity}

To analyze the activation of multiple caspases including caspase-1, $-3,-4,-5,-6,-7,-8$, and -9 , the Muse ${ }^{\mathrm{TM}}$ Multi-caspase Kit (MCH100109, EMD Millipore) was used according to the manufacturer's instruction. Briefly, cells treated with PT were harvested and washed with $1 \times$ caspase buffer. The washed cells were resuspended in Muse ${ }^{\mathrm{TM}}$ Multi-caspase Reagent working solution for $30 \mathrm{~min}$ at $37^{\circ} \mathrm{C}$. Then, the cells were incubated for 5 min after adding Muse ${ }^{\mathrm{TM}}$ Caspase 7-AAD working solution. The activation of multiple caspases was analyzed by flow cytometry using a Muse ${ }^{\mathrm{TM}}$ Cell Analyzer.

\section{Western blot assay}

To lyse the cells, the harvested cells were washed with cold $1 \times$ PBS, and then resuspended for sonication in RIPA buffer (iNtRON Biotechnology, Seongnam, Korea). A Bio-Rad DC Protein Assay kit was used to determine the protein concentration of the cell lysates (Bio-Rad, Hercules, CA, USA). Then, equal amounts of protein samples were resolved by $6-15 \%$ sodium dodecyl sulfate-polyacrylamide gel electrophoresis. The resolved proteins were transferred to poly-vinylidene fluoride membranes (EMD Millipore). The membranes were blocked in $3 \%$ or $5 \%$ skim milk in $1 \times$ PBST (PBS containing $0.1 \%$ Tween $20)$ for $2 \mathrm{~h}$ at RT and washed with PBST. The respective pri- mary antibodies were used to probe the membranes. The dilution of the primary antibodies was $1: 1,000$ in $1 \times$ PBST, and the incubation was either $2 \mathrm{~h}$ at RT, or overnight at $4^{\circ} \mathrm{C}$. Next, the membranes were washed with PBST three times, then incubated with horseradish peroxidase-conjugated secondary antibodies. The dilution of antibodies was 1:4,000-6,000, and the incubation was $1 \mathrm{~h}$ at RT. Visualization of Western blots was done using Western Blotting Luminol Reagent (Santa Cruz Biotechnology), and detected with ImageQuant LAS 500 (GE Healthcare, Uppsala, Sweden).

\section{Statistical analysis}

All data are presented as the mean \pm standard deviation (SD), of three independent triplicate experiments. One-way ANOVA was used to assess the statistical significance of variation among multiple groups. $p$ values of less than 0.05 $\left.\left({ }^{*}\right), 0.01{ }^{* *}\right)$, and $\left.0.001{ }^{* * *}\right)$ were considered statistically significant.

\section{RESULTS}

\section{PT inhibits cell proliferation and induces apoptosis in HCT116 cells}

To analyze the inhibition of HCT116 cell proliferation by PT (Fig. 1A), the MTT assay was performed. Fig. 1B shows that PT significantly reduced cell viability in dose- and timedependent manners. The $\mathrm{IC}_{50}$ value was $0.23 \mu \mathrm{M}$ for $48 \mathrm{~h}$ of treatment (Fig. 1B). The soft agar assay results indicated that colony formation was inhibited by treatment with increasing concentrations of PT. HCT116 cells treated with $0.1 \mu \mathrm{M}$ PT produced $94.9 \%$ fewer colonies and $87.24 \%$ smaller colonies compared to the untreated control cells (Fig. 1C-1E). We further conducted annexin V-FITC/7-AAD staining, and the cells were analyzed using a Muse ${ }^{\mathrm{TM}}$ Cell Analyzer. As the PT concentration increased from 0 to $0.1,0.2$, and $0.3 \mu \mathrm{M}$, the percentage of apoptotic cells increased from $2.59 \%$ to $18.47 \%$, $28.87 \%$, and $65.90 \%$, respectively (Fig. $1 \mathrm{~F}, 1 \mathrm{G}$ ). These results suggested that HCT116 cell apoptosis was induced by PT treatment.

\section{PT induces G2/M cell cycle arrest in HCT116 cells}

We examined the effect of PT on the cell cycle phase populations of HCT116 cells using the Muse ${ }^{\mathrm{TM}}$ Cell Analyzer. The percentage of cells in the sub-G1 phase increased from $5.33 \%$ in the DMSO-treated group to $19.93 \%, 34.67 \%$, and $49.27 \%$ after treatment with $0.1,0.2$, and $0.3 \mu \mathrm{M} \mathrm{PT}$, respectively (Fig. $2 \mathrm{~A}, 2 \mathrm{~B})$. Compared to the control cells, the percentage of HCT116 cells in the G2/M phase increased as the treatment concentration of PT increased, whereas the G0/G1 phases were decreased, and the $S$ phases were unaffected by PT treatment (Fig. 2A, 2C) Furthermore, we conducted Western blots to assess the expression of G2/M phase-related proteins in HCT116 cells. After PT treatment, cyclin B1 and cdc2 were downregulated in HCT116 cells, and simultaneously p27 and p21 were upregulated in a concentration-dependent manner compared to the control group (Fig. 2D). These observations suggest that the PT-mediated decrease in cell viability were accompanied by $\mathrm{G} 2 / \mathrm{M}$ cell cycle arrest.

PT increases intracellular ROS production in HCT116 cells Our next goal was to investigate whether the PT-induced 
A<smiles></smiles>

Podophyllotoxin (PT)

C

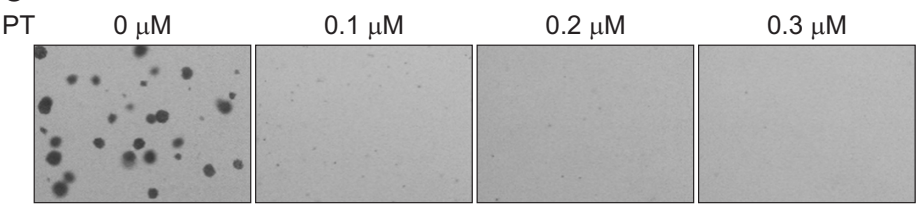

$\mathbf{F}$
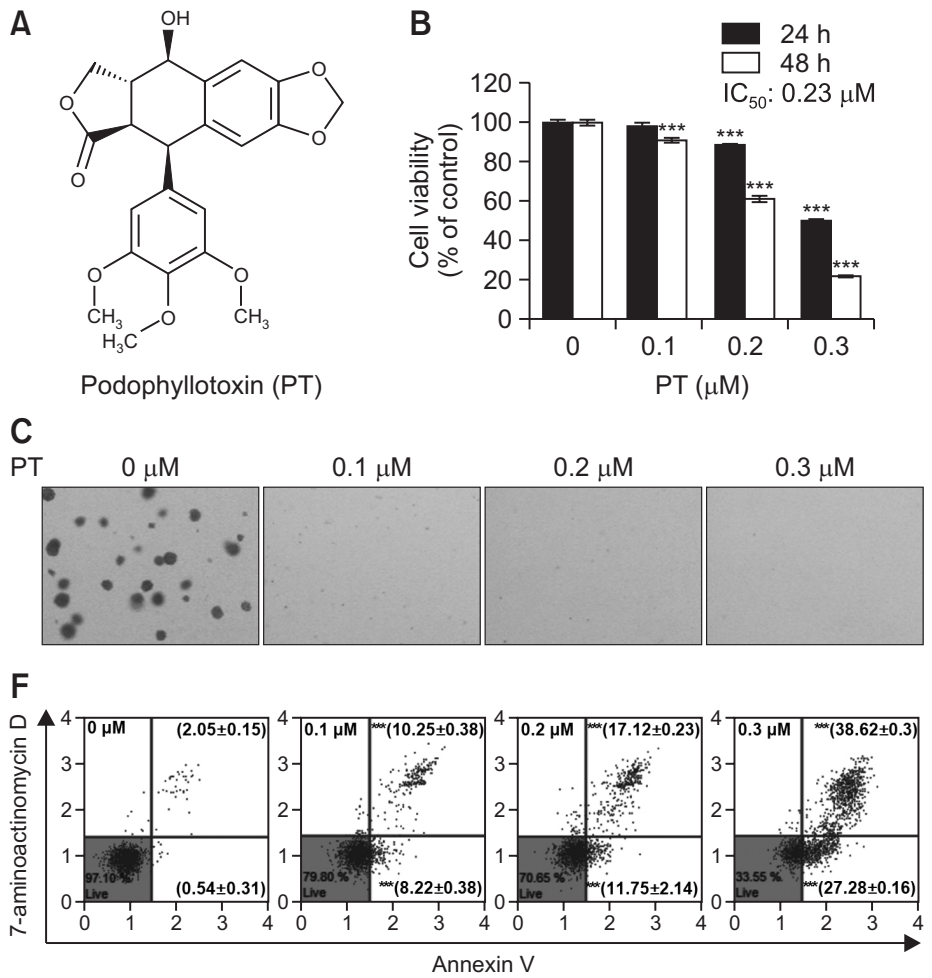

D

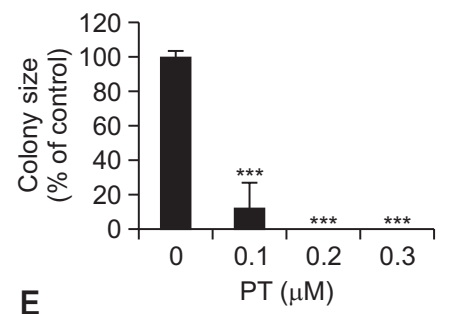

E
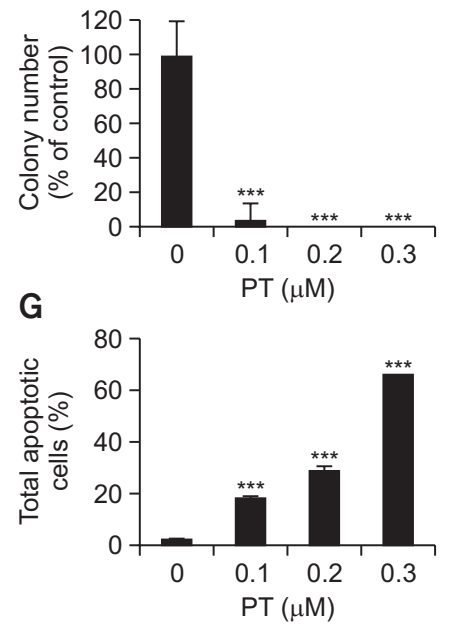

Fig. 1. Effect of PT on cell proliferation and apoptosis. (A) Chemical structure of podophyllotoxin. (B) Cells were treated with increasing concentrations of PT $(0,0.1,0.2$, and $0.3 \mu \mathrm{M})$ for 24 or $48 \mathrm{~h}$ and cell viability was determined by the MTT assay. (C-E) HCT116 cells were exposed to PT and colony formation was determined by the soft agar assay. After 7 days of culture, the number and size of the colonies were analyzed using a microscope. $(F, G)$ The cells were analyzed by the Muse ${ }^{\mathrm{TM}}$ Cell Analyzer using annexin V-FITC/7-AAD staining. $\mathrm{HTC} 116$ cells were treated with $0,0.1,0.2$, and $0.3 \mu \mathrm{M}$ of PT for $48 \mathrm{~h}$. Dot plot graphs representing the percentage of live (annexin-V-/7$A A D-$ ), early apoptotic (annexin- $V+/ 7-A A D-)$, late apoptotic (annexin- $V+/ 7-A A D+$ ), and necrotic (annexin- $V-/ 7-A A D+$ ) cells. The values are expressed as the mean \pm SD of triplicate determinations from three independent experiments. ${ }^{* * *} p<0.001$ vs. the control group.

apoptosis was mediated by the generation of intracellular ROS. We measured the production of ROS in HCT116 cells after treatment with $\mathrm{PT}$ for $48 \mathrm{~h}$ using a Muse ${ }^{\mathrm{TM}}$ Oxidative Stress Kit. The percentage of ROS + HCT116 cells increased from $5.88 \%$ to $42.59 \%$, after treatment with $0 \mu \mathrm{M}$ to $0.3 \mu \mathrm{M}$ PT (Fig. 3A, 3B). HCT116 cells were co-treated with $0.3 \mu \mathrm{M}$ PT and $4 \mathrm{mM}$ ROS scavenger (NAC) and the cell viability was analyzed by the MTT assay. As shown in Fig. 3C, the reduction in cell viability induced by treatment with PT alone was significantly prevented by addition of NAC, indicating that ROS production was essential for PT-induced HCT116 cell death. To determine if the increase in ROS generation induced ER stress, we examined the expression of ER stress markers by Western blot analysis. In PT-treated HCT116 cells, GRP78 and $\mathrm{CHOP}$ were up-regulated compared to the controls (Fig. 3D). We further evaluated the levels of TRAIL receptor-associated proteins in HCT116 cells following PT treatment. Treatment with PT resulted in the up-regulation of DR5 and DR4 in concentration-dependent manners (Fig. 3D). These results demonstrated that PT induced ER stress-mediated apoptosis in HCT116 cells via the upregulation of ROS.

\section{PT regulates p38 MAPK signaling in HCT116 cells}

We performed Western blotting of HCT116 cells to examine whether PT could regulate activation of the p38 signaling pathway. The treatment of HCT116 cells with $0.3 \mu \mathrm{M}$ PT increased p38 MAPK phosphorylation (Fig. 4A). To confirm the role of p38 MAPK in mediating PT-induced apoptosis, HCT116 cells were pretreated with $5 \mu \mathrm{M}$ SB203580 (p38 MAPK inhibitor) for $3 \mathrm{~h}$ before treatment with $0.3 \mu \mathrm{M}$ of PT for $48 \mathrm{~h}$. As shown in Fig. 4B, the apoptotic effect of PT was prevented by pretreatment HCT116 cells with the p38 inhibitor. Taken together, these results suggest that the p38 signaling pathway was a key regulator of PT-induced apoptosis in HCT116 cells.

\section{PT reduces mitochondrial membrane potential and induces caspase-dependent apoptosis in HCT116 cells}

To see whether PT treatment resulted in the loss of MMP, we monitored the membrane potential of mitochondria after treatment with PT. We observed a concentration-dependent increase in the percentage of all depolarized cells from 6.03 $\pm 0.30 \%$ in the controls to $7.28 \pm 0.28 \%, 26.00 \pm 0.72 \%$, and $44.65 \pm 1.01 \%$ in following treatment with $0.1,0.2$, and $0.3 \mu \mathrm{M}$ PT, respectively (Fig. 5A, 5B). To investigate the expression levels of apoptotic pathway-related proteins after PT treatment, we performed Western blot assay. When HCT116 cells were incubated with $\mathrm{PT}$, the expression of Bid, Bcl-xl and $\mathrm{Mcl}-1$ was reduced in a dose-dependent manner, whereas Bad expression was increased in a dose-dependent manner. Also, treatment with PT resulted in the increased expression levels of Apaf-1 and the cleavage of pro-caspase 3 in HCT116 cells (Fig. $5 \mathrm{C})$. To determine whether the PT-induced apoptosis could be 
A
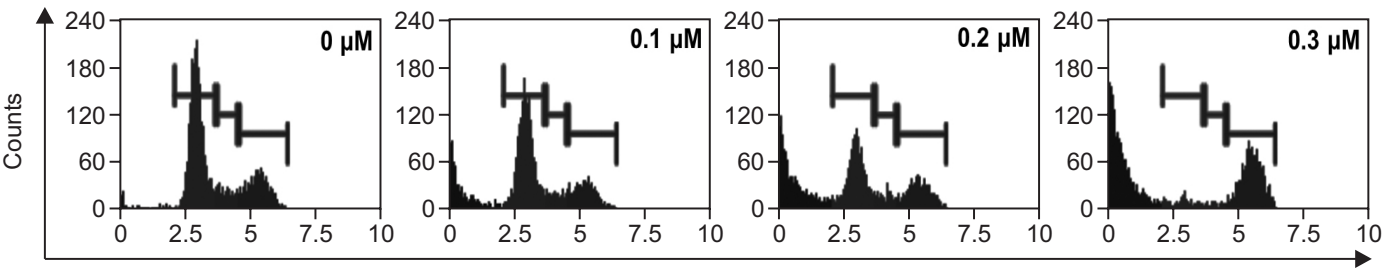

DNA content index

B

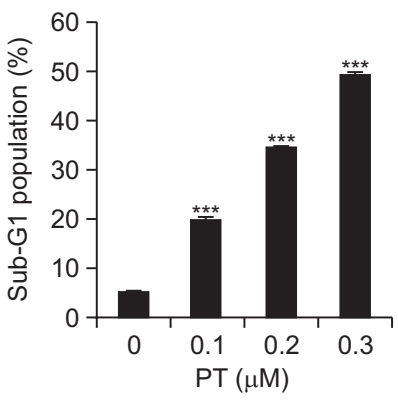

C

Go/G1 $\square \mathrm{S} \square \mathrm{G} 2 / \mathrm{M}$

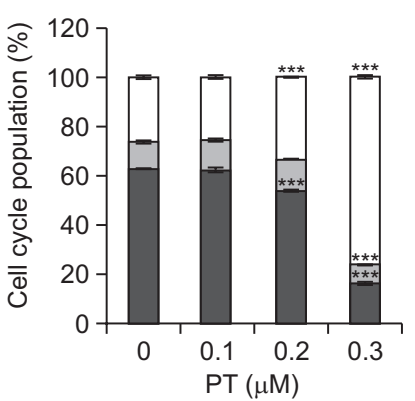

D

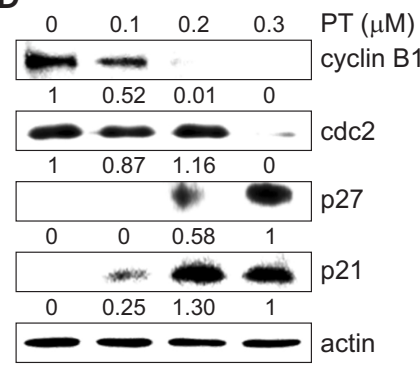

Fig. 2. Effect of PT on cell cycle distribution in HCT116 cells. HCT116 cells were treated with $0,0.1,0.2$, and $0.3 \mu \mathrm{M} P T$ for $48 \mathrm{~h}$. (A) Cellcycle analysis was conducted by the Muse ${ }^{\mathrm{TM}}$ Cell Analyzer using propidium iodide (PI) staining. (B, C) The bar graphs represent the mean \pm SD of triplicate determinations from three separate experiments. Statistical notations for differences in cell cycle populations compared to controls: ${ }^{* * *} p<0.001$. (D) The expression level of cell cycle-related proteins in HCT116 cells was analyzed by Western blotting assays. Actin was used as the loading control.

A
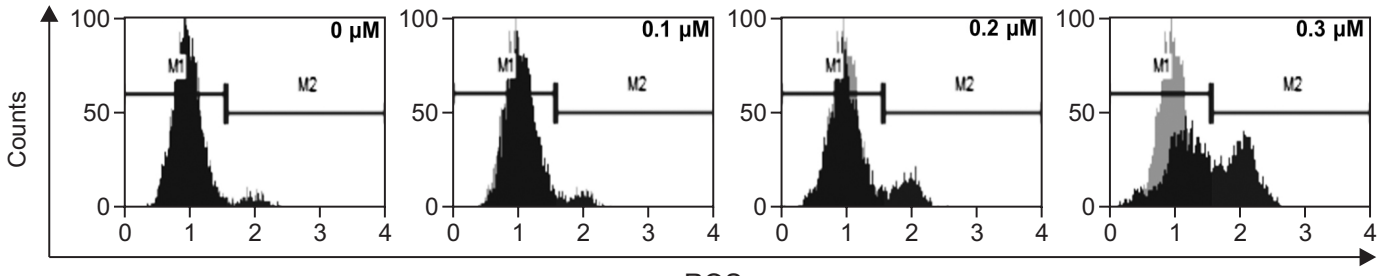

ROS

B

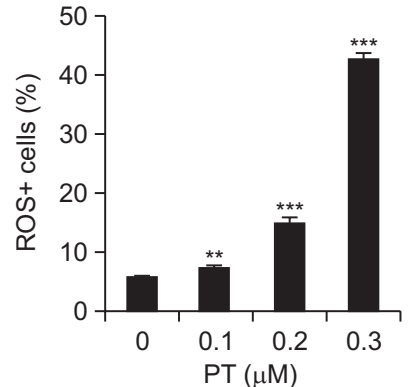

C

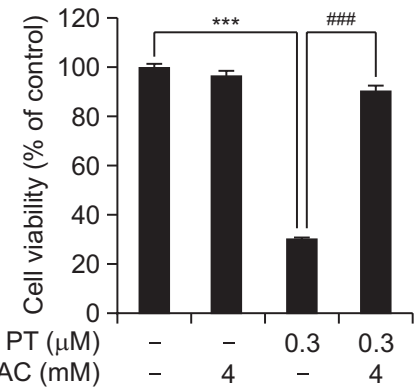

D

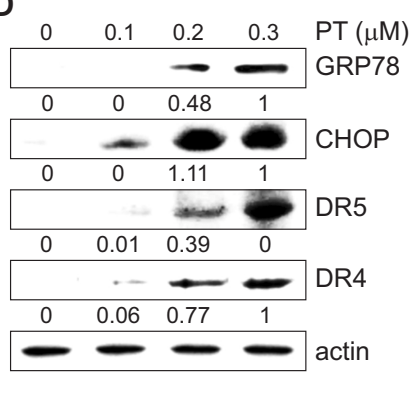

Fig. 3. Effect of PT on ROS production and ROS-mediated apoptosis. HCT116 cells were exposed to PT $(0,0.1,0.2$, and $0.3 \mu \mathrm{M})$ for $48 \mathrm{~h}$ and strained with the Muse ${ }^{\mathrm{TM}}$ Oxidative Stress Kit. (A) The plots represent the population of ROS- cells (M1) and ROS+ cells (M2). (B) Fluorescence intensity data are shown as the mean \pm SD of three independent samples. (C) HCT116 cells were pre-treated with or without 4 mM $\mathrm{NAC}$ for $3 \mathrm{~h}$, then incubated with $0.3 \mu \mathrm{M}$ PT for $48 \mathrm{~h}$. Cell viability was determined by the MTT assay. The data are expressed as the mean \pm SD from three replicates of three independent experiments. ${ }^{* *} p<0.01$ and ${ }^{* * *} p<0.001$ compared to the control group and ${ }^{\# \# \#} p<0.001$ compared to the PT-treated group. (D) Western blot analysis of GRP78, CHOP, DR4 and DR5 proteins from cells incubated with different concentrations of PT for $48 \mathrm{~h}$. Actin was used as the loading control. 
A

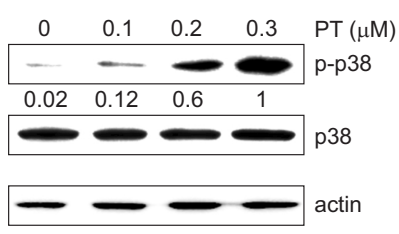

B

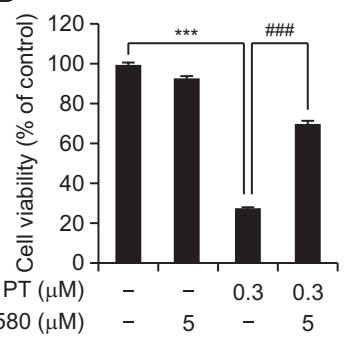

Fig. 4. Effect of PT on p38 MAPK signaling pathways in HCT116 cells. (A) HCT116 cells were treated with $0,0.1,0.2$, and $0.3 \mu \mathrm{M}$ PT for $48 \mathrm{~h}$, and the phosphorylation of p38 (p-p38) was evaluated by Western blots. Actin was used as the protein loading control. (B) HCT 116 cells were untreated or treated with PT for $48 \mathrm{~h}$ in the absence or presence of a p38 inhibitor for $3 \mathrm{~h}$. Cell viability was assessed using the MTT assay. All experiments were performed in triplicate and the data represent the mean \pm SD. ${ }^{* * *} p<0.001$ to controls, ${ }^{\#} p<0.001$ compared to PT-treated cells.

associated with multi-caspases, caspase activity was analyzed by the Muse ${ }^{\mathrm{TM}}$ Multi-caspase Kit. Incubation of HCT116 cells with $0.1,0.2$, and $0.3 \mu \mathrm{M}$ PT for $48 \mathrm{~h}$ increased the percentage of cells with caspase activity in the untreated group from 6.15 $\pm 0.38 \%$ to $8.62 \pm 1.12 \%, 31.61 \pm 2.56$, and $48.04 \pm 1.56 \%$, respectively (Fig. 5D, 5E). Furthermore, HCT116 cells were pretreated with Z-VAD-FMK $(4 \mu \mathrm{M})$, a pan-caspase inhibitor, for $3 \mathrm{~h}$, and then exposed to $0.3 \mu \mathrm{M}$ PT for $48 \mathrm{~h}$. Cell viability decreases were significantly prevented in PT and Z-VAD-FMK treatment groups, compared to the viability in the PT-treated groups of HCT116 cells (Fig. 5F) These results suggested that PT induced the apoptosis of colon cancer cells via a caspasedependent pathway.

\section{DISCUSSION}

PT is a phytochemical exerting various biological activities, and multiple studies have demonstrated the anticancer activity of PT and its derivatives in cancer cell lines and animal
A

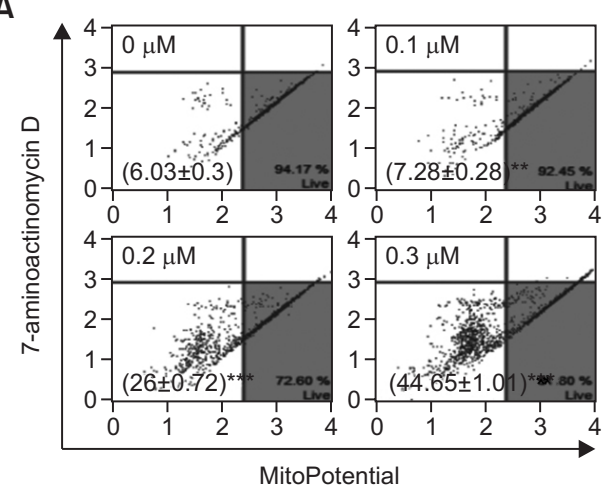

D

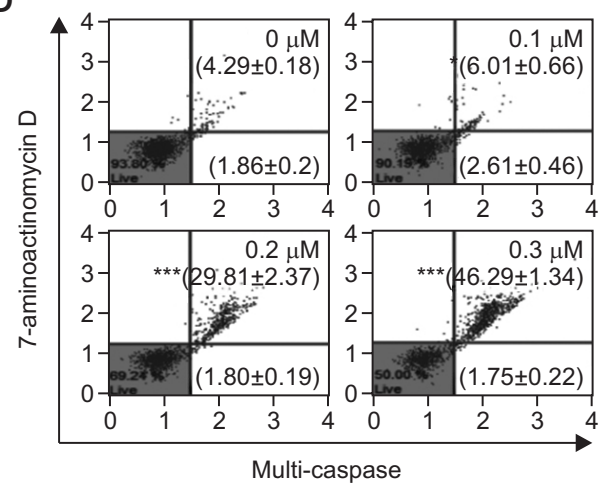

B

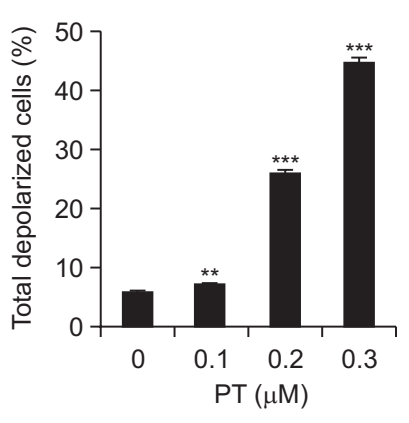

E

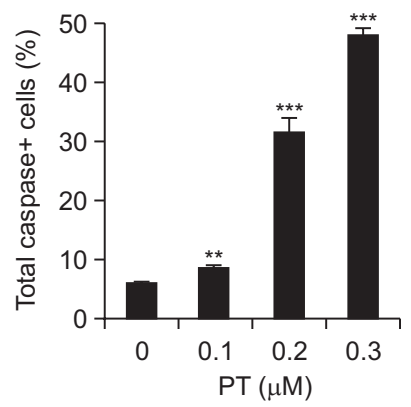

C

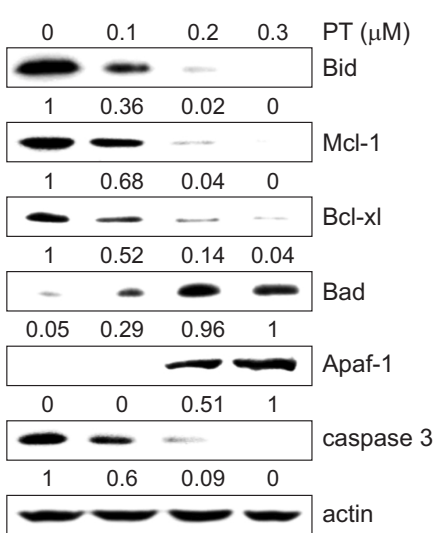

$\mathbf{F}$

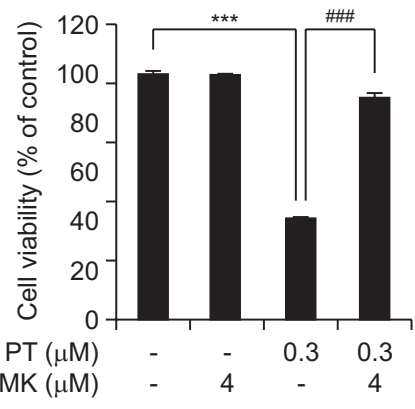

Fig. 5. Effect of PT on MMP disruption and caspase activity in HCT116 cells. HCT116 cells were treated with $0,0.1,0.2$, and $0.3 \mu \mathrm{M}$ of $\mathrm{PT}$ for $48 \mathrm{~h}$. (A) MMP was assessed by staining with MitoPotential dye and 7-aminoactinomycin D and was measured by the Muse ${ }^{\mathrm{TM}}$ Cell Analyzer. Top left quadrant: depolarized dead cells; top right quadrant: dead cells; lower left quadrant: depolarized live cells; lower right quadrant: live cells with intact mitochondrial membrane potential. (B) Bar charts depicting the total percentage of all depolarized cells. (C) Western blots of the expression of Bid, Bcl-xl, Mcl-1, Bad, Apaf-1, and caspase 3 in HCT116 cells following treatment with PT for 48 h. Actin served as the loading control. (D) Multi-caspases (caspase-1, -3, -4, -5, -6, -7, -8, and -9) were evaluated using the Muse ${ }^{\mathrm{TM}}$ MultiCaspase Kit. Each quadrant represents live cells, caspase-positive cells, caspase-positive/dead cells and dead cells in the counter-clockwise direction. (E) Histograms showing the average percentage of all cells with multi-caspase activation. (F) HCT116 cells were pretreated with ZVAD-FMK (a pan-caspase inhibitor) for $3 \mathrm{~h}$, followed by treatment with PT for $48 \mathrm{~h}$. Cell viability was measured by the MTT assay. The data are presented as the means \pm SD $(n=3)$. ${ }^{*} p<0.05,{ }^{* *} p<0.01$, and ${ }^{* * *} p<0.001$ compared to the control group; ${ }^{\# \#} p<0.001$ compared to PT alone. 
experiments. PT inhibited the growth of a human breast cancer cell line by $50 \%$ at $1 \mathrm{nM}$ (Chattopadhyay et al., 2004). Nanoliposomes of PT inhibited the growth of liver cancer in mice (Zhang et al., 2006). Whereas the anticancer activity of PT is mainly from the inhibition of DNA topoisomerase II and microtubule assembly (Sathish et al., 2018), studies are revealing more molecular targets of PT. PT was shown to inhibit the growth of triple-negative breast cancer cells such as MDAMB-231 (Zhang et al., 2020). We recently reported that PT inhibited the growth of gefitinib-resistant non-small cell lung cancer cells by targeting c-MET (Oh et al., 2021). Moreover, the derivatization of PT allowed for the synthesis of versatile analogs with potent anticancer activity (Yu et al., 2017). Thus, PT is one of the most attractive natural compounds considering its biological activities and the possibility of derivatization. This was the first report on the anticancer activity of PT in human colorectal cancer HCT116 cells. Our MTT assay results indicated that the proliferation of HCT116 cells was inhibited by PT in a dose-dependent manner. Anchorage-independent colony formation assessed by soft agar assay also decreased upon PT treatment.

Apoptosis is characterized by morphological and biological changes including chromatin condensation followed by DNA degradation (Khan et al., 2014), and it can be an attractive target for anticancer therapy. Either the induction of apoptosis or the restoration of apoptotic signaling pathways can result in apoptosis in cancer cells, and many anticancer strategies target apoptosis (Wong, 2011). As we confirmed that PT had a cytotoxic effect on HCT116 cells, we tested whether PT induced the apoptosis of HCT116 cells. The annexin V/7-AAD double staining assay revealed that the rate of apoptosis increased significantly $48 \mathrm{~h}$ after treatment with PT. To further investigate the inhibition of cell growth caused by PT treatment, we evaluated the effect of PT on cell cycle distribution in HCT116 cells. Cell cycle analysis confirmed that PT strongly induced the accumulation of mitotic cells in HCT116 cells. Most of the HCT116 cells were arrested in the G2/M phase and the percentage of HCT116 cells in the sub-G1 phase, evidence of DNA degradation, was significantly increased after PT treatment in a concentration-dependent manner compared to the controls. To investigate the underlying molecular mechanism of cell cycle arrest induced by PT, we focused on cyclins and cyclin-dependent kinases (CDKs). Cyclins and CDKs form specific complexes that are critical regulators cell cycle progression and their activation (Bai et al., 2017). The cyclin B1/CDK1 complex plays an important role at the G2/M transition in response to DNA damage (Shang et al., 2015). CDK/cyclin complexes are regulated by the binding of CDKinteracting protein/kinase inhibitory proteins, of which p21 and p27 specifically bind with and inhibit cdc2/cyclin B1 complexes (Craig et al., 1997; Dash and El-Deiry, 2005). In the present study, we found that the treatment of HCT116 cells with PT substantially suppressed the protein levels of cyclin B1, cdc2, but increased the protein levels of p21and p27.

ROS are involved with diverse cellular functions, and low to moderate levels of ROS seem essential for cell viability (Liu et al., 2014). However, the excessive production of ROS results in the death of cancer cells through the activation of apoptotic pathways (Liou and Storz, 2010; Li et al., 2017). Our previous study suggested that PT induced apoptosis in non-small cell lung cancers via a ROS-dependent mechanism (Oh et al., 2021). In this study, we found that PT increased cellular
ROS levels, and the PT-induced apoptotic effects were inhibited by NAC, an ROS scavenger. These results suggest that the increase in the generation of ROS mediated PT-induced apoptosis. Even though it awaits further study for an understanding of how PT induces the increase in the generation of ROS, it is suspected that the overproduction of ROS by NADPH oxidase (NOX) (Weyemi et al., 2010) and down regulation of antioxidant defense mechanisms would be involved (Chio and Tuveson, 2017). In this study, we monitored the level of ROS using Muse ${ }^{\mathrm{TM}}$ Oxidative Stress kit, measuring the level of total ROS in cytoplasm. Further study using pharmacological inhibitors would reveal the molecular source of the ROS such as NADPH oxidase, mitochondrial respiratory chain complex I, xanthine oxidase, and nitric oxide synthase (Chae et al., 2020). Elevated ROS levels are implicated in ER stress, and the well-known biomarkers of ER stress are GRP78 and CHOP (Zhao et al., 2015; Kim et al., 2020). CHOP is a transcription factor of DR4 and DR5 that leads to the extrinsic apoptotic pathway (Hu et al., 2019). Our data showed that PT activated ER stress-induced apoptosis through the production of ROS, as evidenced by the upregulation of GRP78, CHOP, DR4, and DR5 expression in HCT116 cells.

In response to oxidative stress in the ER, the MAPK signaling pathway can be activated (Darling and Cook, 2014). JNK and p38 belong to the MAPK pathway and can be activated through a series of phosphorylations in the signaling pathway when ROS generation increases. The activation of MAPK in this context seems to be dependent upon elevated levels of ROS, as an antioxidant such as NAC could inhibit activation of the MAPK signaling pathway (Son et al., 2011). In this study, we observed the upregulation of ROS levels induced by PT (Fig. 3A, 3B) and the increased phosphorylation of p38 MAPK (Fig. 4A). Furthermore, the inhibitor of p38, SB203580, strongly prevented the cytotoxicity of PT (Fig. 4B). We suspect that the increased generation of ROS may act upstream of the p38 MAPK signaling pathway in HCT116 cells treated with PT. P38 MAPK signaling pathway regulates the transcription and posttranslational modification of several proteins involved with apoptosis including caspases and $\mathrm{Bcl}-2$ family proteins (Wagner and Nebreda, 2009). The signal transducer and activator of transcription 3 (STAT3) is one of the substrates of p38 MAPK, and the activity of STAT3 is modulated by oxidative stress (Chun et al., 2020). Further study would enlighten how p38 MAPK signaling mediates the apoptotic process in colorectal cancer cells.

ER stress can promote the opening of mitochondrial permeability transition pore and induce the loss of MMP, leading to the intrinsic apoptotic pathway (Zeeshan et al., 2016; Richa et al., 2020). Our data showed that PT induced the loss of MMP in HCT116 cells (Fig. 5A, 5B). In addition, we observed a shift in the balance between pro-apoptotic and anti-apoptotic $\mathrm{Bcl}-2$ proteins (Lee et al., 2019). Western blot analysis showed that the level of anti-apoptotic proteins (Bcl-xl and $\mathrm{Mcl}-1$ ) was decreased by PT treatment, whereas the level of pro-apoptotic protein Bad increased (Fig. 5C). Moreover, increased levels of Apaf-1 and the cleavage of procaspase 3 indicated that PT-induced apoptosis was mediated through the intrinsic apoptotic pathway (Fig. 5C). Caspases have a central role in mediating cell death and inflammation (Van Opdenbosch and Lamkanfi, 2019). Caspase activity assessed by the multi-caspase assay revealed that PT induced the activation of multi-caspase, implying the involvement of caspases 
in the PT-induced apoptosis of HCT116 cells (Fig. 5D, 5E). In the presence of the pan-caspase inhibitor (Z-VAD-FMK), cell viability decrease was somewhat prevented in HCT116 cells treated with PT (Fig. 5F), indicating that PT-induced apoptosis was dependent upon caspases. In this study, PT induced the loss of MMP and the activation of multi-caspases, and these accompanied the increase in ROS generation. It is plausible that ROS generation was directly or indirectly related to the loss of MMP and multi-caspase activation.

In conclusion, PT induced G2/M phase cell cycle arrest, the loss of MMP, the activation of multi-caspases, the p38 MAPK signaling pathway, and apoptosis in HCT116 cells. Considering that the anticancer effect of PT was prevented by NAC, increases in ROS generation mediated the anticancer activity of PT. Our results demonstrated that PT induced the apoptosis of HCT116 cells through the upregulation of ROS. More molecular targets of PT are emerging, and the structural versatility of PT derivatives could lead to the development of potential anticancer therapeutics.

\section{CONFLICT OF INTEREST}

The authors have no financial conflicts of interest to declare.

\section{ACKNOWLEDGMENTS}

We greatly appreciated using the Convergence Research Laboratory (established by the MNU Innovation Support Project in 2019) to conduct this research. This research was funded by the Basic Science Research Program of National Research Foundation Korea, grant number 2019R1A2C1005899.

\section{REFERENCES}

Bai, J., Li, Y. and Zhang, G. (2017) Cell cycle regulation and anticancer drug discovery. Cancer Biol. Med. 14, 348-362.

Canel, C., Moraes, R. M., Dayan, F. E. and Ferreira, D. (2000) Podophyllotoxin. Phytochemistry 54, 115-120.

Chae, I. G., Song, N. Y., Kim, D. H., Lee, M. Y., Park, J. M. and Chun, K. S. (2020) Thymoquinone induces apoptosis of human renal carcinoma Caki-1 cells by inhibiting JAK2/STAT3 through pro-oxidant effect. Food Chem. Toxicol. 139, 111253.

Chattopadhyay, S., Bisaria, V. S., Panda, A. K. and Srivastava, A. K. (2004) Cytotoxicity of in vitro produced podophyllotoxin from Podophyllum hexandrum on human cancer cell line. Nat. Prod. Res. 18, 51-57.

Chio, I. I. C. and Tuveson, D. A. (2017) ROS in cancer: the burning question. Trends Mol. Med. 23, 411-429.

Choi, B. H., Kim, J. M. and Kwak, M. K. (2021) The multifaceted role of NRF2 in cancer progression and cancer stem cells maintenance. Arch. Pharm. Res. 44, 263-280.

Chun, K. S., Jang, J. H. and Kim, D. H. (2020) Perspectives regarding the intersections between STAT3 and oxidative metabolism in cancer. Cells 9, 2202.

Craig, C., Wersto, R., Kim, M., Ohri, E., Li, Z., Katayose, D., Lee, S. J., Trepel, J., Cowan, K. and Seth, P. (1997) A recombinant adenovirus expressing p27Kip1 induces cell cycle arrest and loss of cyclin-Cdk activity in human breast cancer cells. Oncogene 14, 2283-2289.

Darling, N. J. and Cook, S. J. (2014) The role of MAPK signalling pathways in the response to endoplasmic reticulum stress. Biochim. Biophys. Acta 1843, 2150-2163.
Dash, B. C. and El-Deiry, W. S. (2005) Phosphorylation of p21 in G2/M promotes cyclin B-Cdc2 kinase activity. Mol. Cell. Biol. 25, 33643387.

Deng, X., Ruvolo, P., Carr, B. and May, W. S., Jr. (2000) Survival function of ERK1/2 as IL-3-activated, staurosporine-resistant Bcl2 kinases. Proc. Natl. Acad. Sci. U.S.A. 97, 1578-1583.

Hande, K. R. (1998) Etoposide: four decades of development of a topoisomerase II inhibitor. Eur. J. Cancer 34, 1514-1521.

Hu, H., Tian, M., Ding, C. and Yu, S. (2019) The C/EBP homologous protein (CHOP) transcription factor functions in endoplasmic reticulum stress-induced apoptosis and microbial infection. Front. Immunol. 9, 3083.

Hu, L. L., Liao, B. Y., Wei, J. X., Ling, Y. L., Wei, Y. X., Liu, Z. L., Luo, X. Q. and Wang, J. L. (2020) Podophyllotoxin exposure causes spindle defects and DNA damage-induced apoptosis in mouse fertilized oocytes and early embryos. Front. Cell Dev. Biol. 8, 600521.

Khan, K. H., Blanco-Codesido, M. and Molife, L. R. (2014) Cancer therapeutics: targeting the apoptotic pathway. Crit. Rev. Oncol. Hematol. 90, 200-219.

Kim, T. W., Hong, D. W. and Hong, S. H. (2020) CB13, a novel PPARgamma ligand, overcomes radio-resistance via ROS generation and ER stress in human non-small cell lung cancer. Cell Death Dis. 11, 848.

Ko, Y. H., Kim, S. K., Kwon, S. H., Seo, J. Y., Lee, B. R., Kim, Y. J., Hur, K. H., Kim, S. Y., Lee, S. Y. and Jang, C. G. (2019) 7,8,4'-Trihydroxyisoflavone, a metabolized product of daidzein, attenuates 6-hydroxydopamine-induced neurotoxicity in SH-SY5Y cells. Biomol. Ther. (Seoul) 27, 363-372.

Lee, Y. J., Kim, W. I., Kim, S. Y., Cho, S. W., Nam, H. S., Lee, S. H. and Cho, M. K. (2019) Flavonoid morin inhibits proliferation and induces apoptosis of melanoma cells by regulating reactive oxygen species, Sp1 and Mcl-1. Arch. Pharm. Res. 42, 531-542.

$\mathrm{Li}, \mathrm{A}$. X., Sun, M. and Li, X. (2017) Withaferin-A induces apoptosis in osteosarcoma U2OS cell line via generation of ROS and disruption of mitochondrial membrane potential. Eur. Rev. Med. Pharmacol. Sci. 21, 1368-1374.

Liou, G. Y. and Storz, P. (2010) Reactive oxygen species in cancer. Free Radic. Res. 44, 479-496.

Liu, B., Tan, X., Liang, J., Wu, S., Liu, J., Zhang, Q. and Zhu, R. (2014) A reduction in reactive oxygen species contributes to dihydromyricetin-induced apoptosis in human hepatocellular carcinoma cells. Sci. Rep. 4, 7041.

Martindale, J. L. and Holbrook, N. J. (2002) Cellular response to oxidative stress: signaling for suicide and survival. J. Cell. Physiol. 192, 1-15.

Moradi Marjaneh, R., Hassanian, S. M., Ghobadi, N., Ferns, G. A., Karimi, A., Jazayeri, M. H., Nasiri, M., Avan, A. and Khazaei, M. (2018) Targeting the death receptor signaling pathway as a potential therapeutic target in the treatment of colorectal cancer. J. Cell. Physiol. 233, 6538-6549.

Nogueira, V. and Hay, N. (2013) Molecular pathways: reactive oxygen species homeostasis in cancer cells and implications for cancer therapy. Clin. Cancer Res. 19, 4309-4314.

Oh, H. N., Kwak, A. W., Lee, M. H., Kim, E., Yoon, G., Cho, S. S., Liu, K., Chae, J. I. and Shim, J. H. (2021) Targeted inhibition of cMET by podophyllotoxin promotes caspase-dependent apoptosis and suppresses cell growth in gefitinib-resistant non-small cell lung cancer cells. Phytomedicine 80, 153355.

Osone, S., Hosoi, H., Kuwahara, Y., Matsumoto, Y., lehara, T. and Sugimoto, T. (2004) Fenretinide induces sustained-activation of JNK/ p38 MAPK and apoptosis in a reactive oxygen species-dependent manner in neuroblastoma cells. Int. J. Cancer 112, 219-224.

Qiao, L. and Wong, B. C. (2009) Targeting apoptosis as an approach for gastrointestinal cancer therapy. Drug Resist. Updat. 12, 55-64.

Reed, J. C., Cuddy, M., Slabiak, T., Croce, C. M. and Nowell, P. C. (1988) Oncogenic potential of bcl-2 demonstrated by gene transfer. Nature 336, 259-261.

Richa, S., Dey, P., Park, C., Yang, J., Son, J. Y., Park, J. H., Lee, S. H., Ahn, M. Y., Kim, I. S., Moon, H. R. and Kim, H. S. (2020) A new histone deacetylase inhibitor, MHY4381, induces apoptosis via generation of reactive oxygen species in human prostate cancer cells. Biomol. Ther. (Seoul) 28, 184-194. 
Sathish, M., Kavitha, B., Nayak, V. L., Tangella, Y., Ajitha, A., Nekkanti, S., Alarifi, A., Shankaraiah, N., Nagesh, N. and Kamal, A. (2018) Synthesis of podophyllotoxin linked beta-carboline congeners as potential anticancer agents and DNA topoisomerase II inhibitors. Eur. J. Med. Chem. 144, 557-571.

Schieber, M. and Chandel, N. S. (2014) ROS function in redox signaling and oxidative stress. Curr. Biol. 24, R453-R462.

Shah, Z., Gohar, U. F., Jamshed, I., Mushtaq, A., Mukhtar, H., ZiaUi-Haq, M., Toma, S. I., Manea, R., Moga, M. and Popovici, B. (2021) Podophyllotoxin: history, recent advances and future prospects. Biomolecules 11, 603

Shang, Z. F., Tan, W., Liu, X. D., Yu, L., Li, B., Li, M., Song, M., Wang, Y., Xiao, B. B., Zhong, C. G., Guan, H. and Zhou, P. K. (2015) DNA-PKcs negatively regulates cyclin B1 protein stability through facilitating its ubiquitination mediated by Cdh1-APC/C pathway. Int. J. Biol. Sci. 11, 1026-1035.

Siegel, R. L., Miller, K. D. and Jemal, A. (2020) Cancer statistics, 2020. CA Cancer J. Clin. 70, 7-30.

Son, Y., Cheong, Y. K., Kim, N. H., Chung, H. T., Kang, D. G. and Pae, H. O. (2011) Mitogen-activated protein kinases and reactive oxygen species: how can ROS activate MAPK pathways? J. Signal Transduct. 2011, 792639.

Sung, H., Ferlay, J., Siegel, R. L., Laversanne, M., Soerjomataram, I., Jemal, A. and Bray, F. (2021) Global cancer statistics 2020: GLOBOCAN estimates of incidence and mortality worldwide for 36 cancers in 185 countries. CA Cancer J. Clin. 71, 209-249.

Van Opdenbosch, N. and Lamkanfi, M. (2019) Caspases in cell death, inflammation, and disease. Immunity 50, 1352-1364.

Wagner, E. F. and Nebreda, A. R. (2009) Signal integration by JNK and p38 MAPK pathways in cancer development. Nat. Rev. Cancer 9 537-549.
Wang, H., Jiang, D., Liu, J., Ye, S., Xiao, S., Wang, W., Sun, Z., Xie, Y. and Wang, J. (2013) Compound K induces apoptosis of bladder cancer T24 cells via reactive oxygen species-mediated p38 MAPK pathway. Cancer Biother. Radiopharm. 28, 607-614.

Weyemi, U., Caillou, B., Talbot, M., Ameziane-El-Hassani, R., Lacroix, L., Lagent-Chevallier, O., Al Ghuzlan, A., Roos, D., Bidart, J. M., Virion, A., Schlumberger, M. and Dupuy, C. (2010) Intracellular expression of reactive oxygen species-generating NADPH oxidase NOX4 in normal and cancer thyroid tissues. Endocr. Relat. Cancer 17, 27-37.

Wong, R. S. (2011) Apoptosis in cancer: from pathogenesis to treatment. J. Exp. Clin. Cancer Res. 30, 87.

Xie, Y. H., Chen, Y. X. and Fang, J. Y. (2020) Comprehensive review of targeted therapy for colorectal cancer. Signal Transduct. Target. Ther. 5, 22.

Yu, X., Che, Z. and Xu, H. (2017) Recent advances in the chemistry and biology of podophyllotoxins. Chemistry 23, 4467-4526.

Zeeshan, H. M., Lee, G. H., Kim, H. R. and Chae, H. J. (2016) Endoplasmic reticulum stress and associated ROS. Int. J. Mol. Sci. $17,327$.

Zhang, W., Liu, C., Li, J., Liu, R., Zhuang, J., Feng, F., Yao, Y. and Sun, C. (2020) Target analysis and mechanism of podophyllotoxin in the treatment of triple-negative breast cancer. Front. Pharmacol. 11, 1211.

Zhang, X. Y., Ni, J. M. and Qiao, H. (2006) Studies on antitumor effects of podophyllotoxin nanoliposome. Zhongguo Zhong Yao Za Zhi 31, 148-150

Zhao, Y., Yan, Y., Zhao, Z., Li, S. and Yin, J. (2015) The dynamic changes of endoplasmic reticulum stress pathway markers GRP78 and CHOP in the hippocampus of diabetic mice. Brain Res. Bull. 111, 27-35. 